\title{
Operative Behandlung von Wirbelfrakturen - dorsale Instrumentierung
}

\author{
Hubertus Große-Leege, Karl Heinrich Winker
}

\section{Zusammenfassung}

Ende der 80er Jahre fanden dorsale Instrumentierungssysteme weite Verbreitung, die es möglich machten, die Mehrzahl der Frakturen der thorakolumbalen Wirbelsäule operativ zu stabilisieren. Ein Schlüssel zum Erfolg der neuen Systeme ist das Prinzip der Verankerung von Schrauben in den Wirbelpedikeln, den stabilsten Bestandteilen der Wirbelsäule. Sie dienen somit als Verankerungsort für die so genannten Pedikelschrauben, welche über spezielle Backen mit Längsstäben verbunden werden und so ein winkelstabiles Implantat bilden, den Fixateur interne. Dieses Implantat ist heute das Standardimplantat zur Versorgung von Wirbelfrakturen der Brust- und Lendenwirbelsäule und ermöglicht es, Wirbelfrakturen unabhängig von der Frakturklassifikation mit einem Eingriff von dorsal zu versorgen. Der Fixateur interne, den es in zahlreichen Ausführungen gibt, erlaubt zahlreiche Möglichkeiten einer- seits der Reposition, andererseits der Stabilisierung verschiedener Frakturtypen. Es ist möglich, Wirbelkörper wieder aufzurichten, Achsen wiederherzustellen oder unter Zuhilfenahme des Prinzips der Ligamentotaxis in den Spinalkanal einstehende Knochenfragmente zu reponieren. Das System ist nach den Wirkprinzipien der Zuggurtung, der Neutralisation oder der Abstützung einzusetzen, je nach Frakturklassifikation. Aufgrund der sehr guten Knochenverankerung der Schrauben ist die dorsale Stabilisierung bei den meisten operativ zu stabilisierenden Frakturen unverzichtbar, wird aber häufig in Kombination mit ventralen Verfahren eingesetzt. Dieses ist vor allem dann erforderlich, wenn bedingt durch Knochenverlust des ventralen Wirbelkörpers eine hohe Wahrscheinlichkeit eines sekundären Repositionsverlustes besteht. In diesen Fällen folgt das vordere Vorgehen meistens dem dorsalen, kann aber auch zweizeitig vorgenommen werden.

\section{Einleitung}

Die operative Behandlung von Frakturen der thorakolumbalen Wirbelsäule nahm einen großen Aufschwung in den 60er Jahren, mit Verwendung des Disktraktionsinstrumentariums nach Harrington. Paul Harrington führte die operative Behandlung von Skoliosen durch Einbringen metallischer Implantate ein, nachdem es infolge einer KinderlähmungsEpidemie zu zahlreichen lähmungsbedingten Skoliosen im Kinder- und Jugendalter gekommen war [7]. Dieses hin-

OP-JOURNAL 2001; 17: 156-160

(C) Georg Thieme Verlag Stuttgart · New York tere Stabilisierungssystem wurde gegen Ende der 60er Jahre auch zur Stabilisierung von Frakturen genutzt. Die Verankerung der so genannten Harrington-Stäbe erfolgte durch langstreckige Verankerung mit Haken. Das System hatte jedoch zahlreiche Nachteile. Es erforderte eine langstreckige Stabilisierung, das heißt, über 2-3 Segmente oberhalb und unterhalb der Fraktur, war nicht rotationsstabil, die Stangen brachen leicht. Das Prinzip der dorsalen, transpedikulären Schraubenverankerung wurde erstmals in den 80er Jahren von Roy-Camille beschrieben aus der französischen Orthopäden-Schule von Judet [11]. Er hatte den Wirbelpedikel als Verankerungsort für Schrauben entdeckt mit der Erkenntnis, dass der Pedikel der stärkste Teil eines Wirbels ist, somit ideale Voraussetzungen für eine Verankerung bietet. Zunächst wurden Platten verwendet, die die Pedikelschrauben miteinander verbanden. Nachteil dieser Platten war, dass immer noch mehrere benachbarte Wirbelsegmente in das System miteinbezogen werden mussten, um eine ausreichende Stabilisierung zu erreichen. Dieses Miteinbeziehen gesunder Wirbelsegmente ist deshalb nachteilig, als es durch Immobilisation zu sekundären Veränderungen kommt bis hin zur definitiven Versteifung, mit resultierender, eventuell schmerzhafter Bewegungseinschränkung der Wirbelsäule.

Der Durchbruch mit Entwicklung der heute gebräuchlichen Fixateur interne Systeme kam schließlich mit der Entwicklung spezieller Backen, die die winkelstabile Verbindung der Pedikelschrauben über ein Stabsystem möglich machten durch Halten der Schrauben an einem längs angebrachten Stab. Somit wurde eine kurzsteckige Fixierung nur des verletzten Segmentes möglich bis hin zur unisegmentalen Stabilisierung lediglich eines Segmentes. Entwickelt wurde dieses Prinzip durch Magerl mit dem Fixateur externe [10], realisiert dann durch Dick und Kluger [5].

Der Fixateur interne, wie er heute in verschiedenen Ausführungen gebräuchlich ist, gilt als Standardimplantat. Er ermöglicht, alle erforderlichen Repositionsmanöver durchzuführen und stabilisiert nach den bekannten Prinzipien der Neutralisation, Distraktion und Zuggurtung.

Das genannte Stabsystem erlaubt, entlang der Stäbe stufenlos die Repositionsmanöver durchzuführen, indem die bereits in die Pedikel eingedrehten Schrauben in verschiedenen Winkeln mit den Stäben verbunden werden können und dann winkelstabil fixiert über die Backen. 

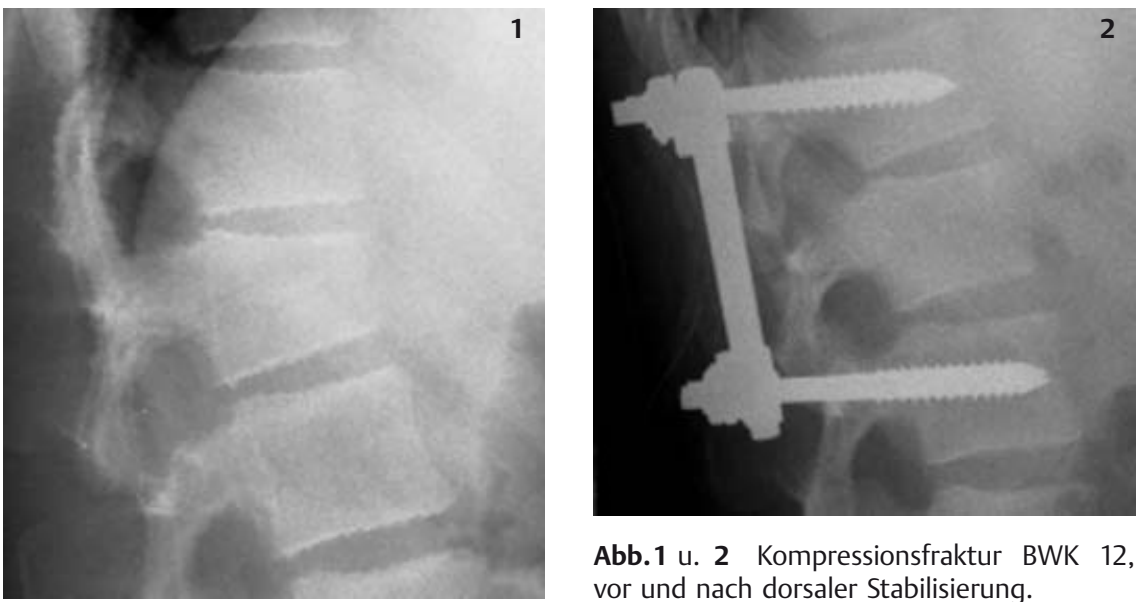

Abb. 1 u. 2 Kompressionsfraktur BWK 12, vor und nach dorsaler Stabilisierung.
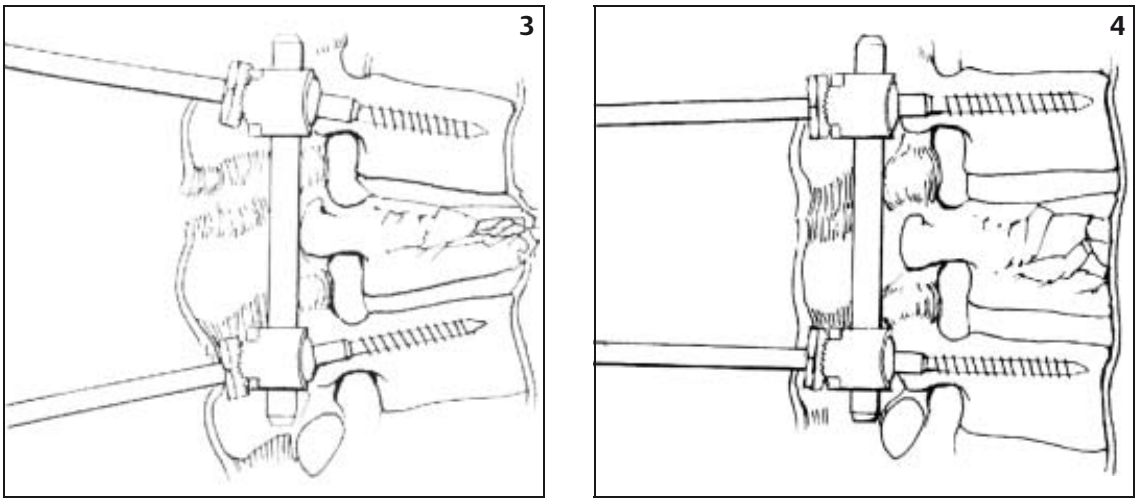

Abb. 3 u. 4 Aufrichtung/Reposition durch Lordosierung.

\section{Instrumentarium}

Eines der häufig benutzten Implantate ist das Pedikelschraubensystem des Universal Spine Systems (USS, Fa. Synthes). Es besteht aus Pedikelschrauben verschiedenen Durchmessers (4-8 mm) sowie verschiedener Längen unter Berücksichtigung des unterschiedlichen anatomischen Aufbaus der Wirbel in verschiedenen Lokalisationen. Die Pedikelhöhe variiert von 12 bis $15 \mathrm{~mm}$, der quere Durchmesser beträgt 5 bis etwa $15 \mathrm{~mm}$. Die maximal mögliche Schraubenlänge variiert zwischen 35 und $52 \mathrm{~cm}$.

Die Stäbe sind in verschiedenen Längen verfügbar $(50-500 \mathrm{~mm})$, um sie der $\mathrm{zu}$ überbrückenden Distanz anpassen zu können.

Neben den entsprechend erforderlichen Schraubenschlüsseln sind Instrumente zur Eröffnung des Pedikels vorhanden, Pfrieme in verschiedenen Stärken, Bohrer, Kirschner-Drähte, Repositionsinstrumente und ein Pin-Schneider, der das Kürzen der Schanz-Schrauben ermöglicht nach Fertigstellen der Montage.

\section{Vorbereitung/Planung}

Wenngleich die Operationsmethode der dorsalen Instrumentierung mit dem Fixateur interne weit verbreitet ist, stellt das Einbringen der Schrauben komplexe Anforderungen an die OP-Planung. Durch falsche Implantatwahl oder Fehllagen der Pedikel kann es zu erheblichen Komplikationen kommen, vor allem Verletzung neurologischer Strukturen mit eventuell irreversiblen Ausfällen.

Die Klassifikation der Fraktur muss anhand der zur Verfügung stehenden Computertomographie der verletzten sowie angrenzenden Wirbelkörper vorgenommen werden, die Anatomie der Pedikel sowie des angrenzenden Spinalkanales dargestellt sein, um Fehllagen der Pedikelschrauben zu vermeiden. Intraoperativ können nur zwei der drei räumlichen Ebenen beurteilt werden, nämlich die sagittale und die koronare. Die axiale Ebene ist nicht darstellbar bei Verwendung des Bildverstärkers.

Festgelegt werden die zu stabilisierenden Segmente, Zahl und Durchmesser der zu verwendenden Pedikelschrauben, eventuell auch das zu verwendende Material (Stahl oder Titan, harte oder weiche Stäbe, Querverbinder).

\section{Lagerung}

Die Bauchlage ist die Standardlagerung für alle dorsalen Eingriffe an der thorakolumbalen Wirbelsäule. Der Verletzte wird dazu nach Intubation im Vorbereitungsraum entweder auf dem OP-Tisch auf den Bauch gedreht oder aber auf einen zweiten Tisch umgelagert, welcher neben den ersten Tisch gefahren wird. In jedem Fall muss ausreichend Personal zur Verfügung stehen, um eine iatrogene Schädigung zu vermeiden. Verwendung findet ein OP-Tisch, welcher im Bereich der zu operierenden Wirbelsäulenabschnitte strahlendurchlässig ist. Zur Gewährleistung einer freien Bauchatmung sowie zur Erreichung eine Hyperlordosierung der thorakolumbalen Wirbelsäule ist das Unterlegen von je einem Kissen unter die Achselhöhlen, einem Kissen unter das Becken sowie von Keilkissen unter die Unterschenkel erforderlich. Die Arme werden auf Armstützen gelagert.

Nach Ankunft im OP-Saal wird die Lagerung nochmals kontrolliert, der Bildwandler gegenüber dem Operateur in Position gebracht und unter Röntgenkontrolle die Ausrichtung der Wirbelsäule in beiden Ebenen überprüft.

Der dargestellte Wirbelsäulenabschnitt sollte der Ausrichtung des Operateurs zum Patienten entsprechend sein. Die Höhe des frakturierten Wirbelkörpers wird markiert auf der Haut.

\section{Operative Technik}

Die Inzision der Haut erfolgt in der Mittellinie entsprechend der Lokalisation des geplanten Implantates. Nach Inzision der Faszie sollte die Muskulatur beiderseits der Dornfortsätze so sparsam wie möglich abgelöst werden, möglichst subperiostal, abgeschoben mit einem Raspartorium. Die Gelenkkapseln werden dabei geschont, die lateral an den Gelenkfortsätzen ansetzende Muskulatur wird erhalten. Blutungen werden bipolar gestillt.

Die Wundspreizer müssen möglichst schonend eingesetzt werden, um Muskelnekrosen zu vermeiden.

Der erste Schritt zur Anlage des Fixateur interne, der in der Regel über ein oder zwei Segmente angelegt wird, ist das Ein- 

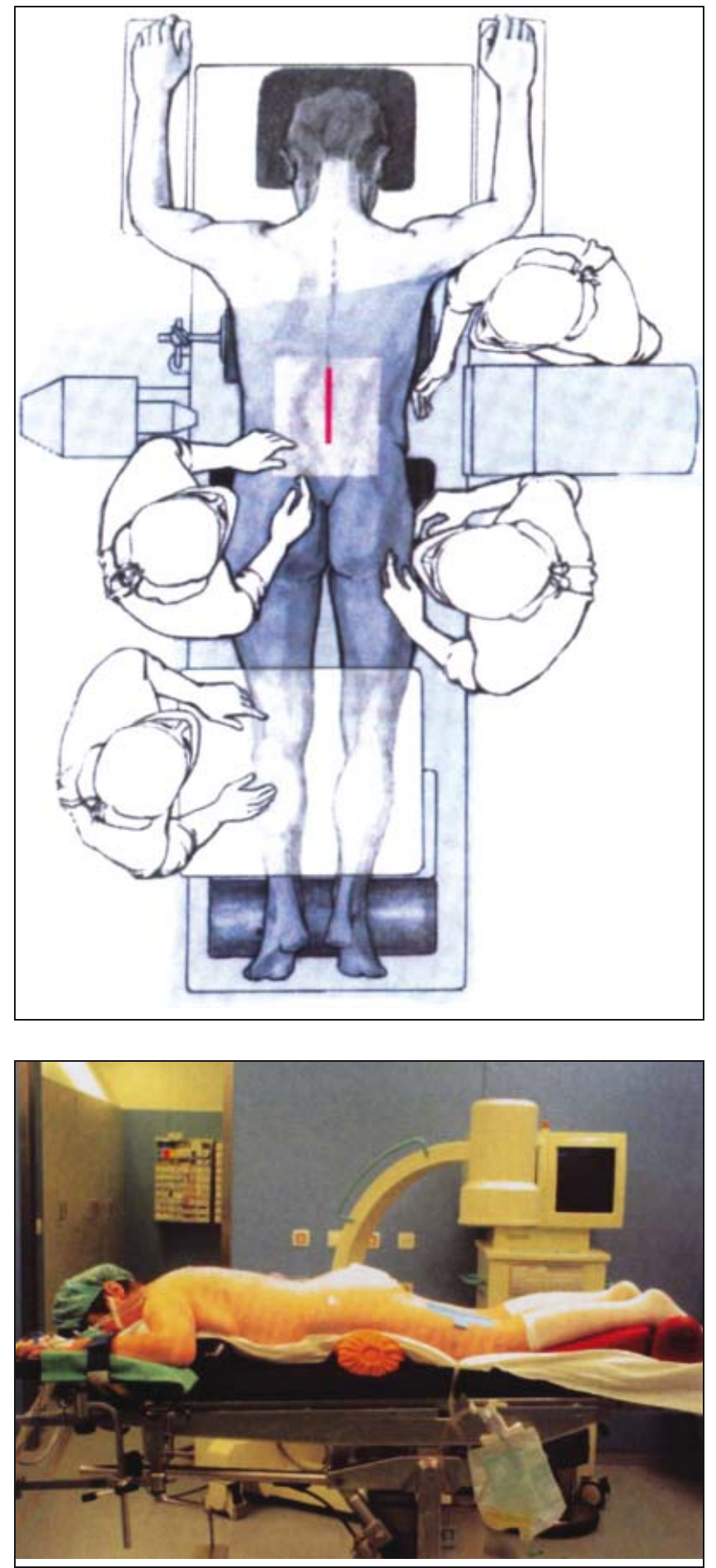

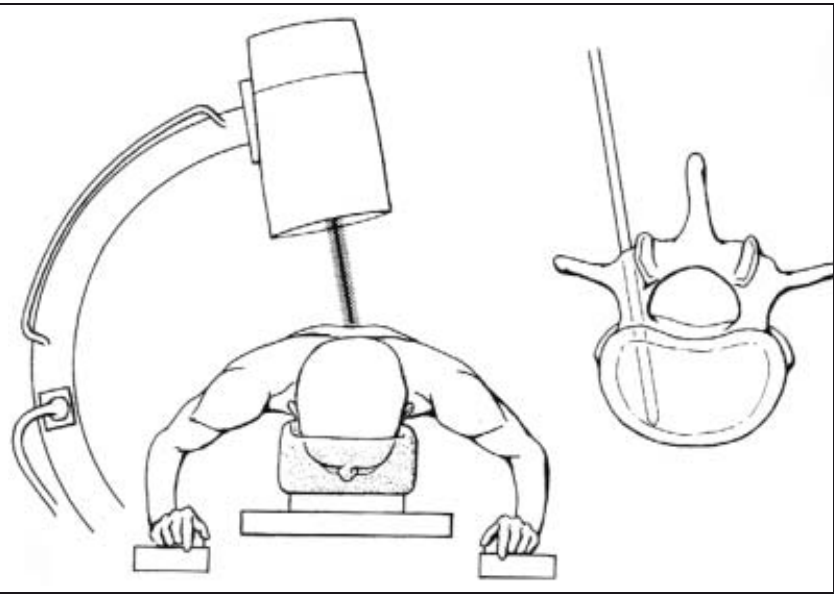

Abb.7 Kontrollmöglichkeit der Schraubenlage unter BV.

Abb. 5 Lagerung, Positionierung BV, Stellung OP-Team.

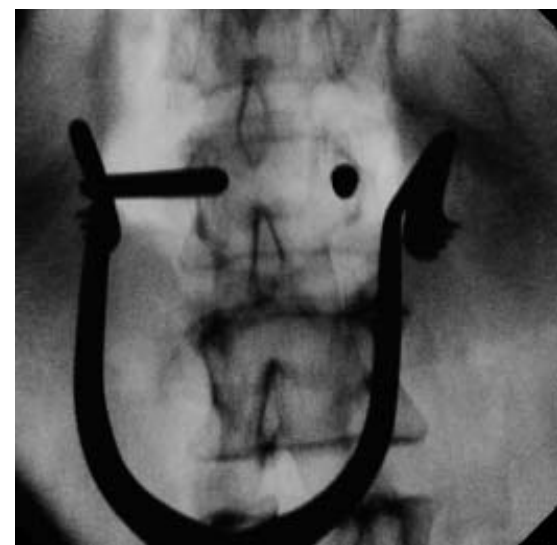

Abb.8 so genannte „Punktprobe“/orthograd getroffene Pedikelschraube unter BV.

Abb. 6 Bauchlagerung auf Kissen im Durchhang.

bringen der Pedikelschrauben. Nachdem die Wirbelkörper im seitlichen Strahlengang sicher identifiziert sind, werden im a.-p.-Strahlengang die Pedikel dargestellt, möglichst orthograd getroffen. Eine vorherige palpatorische Orientierung erleichtert das Auffinden, der Pedikel, vermindert die Strahlenbelastung (Steinmann).
Das Eröffnen der Pedikelkortikalis kann mit einem Pfriem oder einer Ahle erfolgen, oder mit einem Bohrer, kanüliert über einem zuvor unter Durchleuchtung eingebrachten Kirschner-Draht. Wenn gebohrt wird, sollte nur die Kortikalis eröffnet werden, da durch tieferes Aufbohren die Gefahr der Perforation der Pedikelwand gegeben ist.
Anschließend werden die Pedikelschrauben unter Verwendung eines T-Handgriffes eingedreht, die Tiefe der Schrauben im seitlichen Strahlengang kontrolliert.

Um eine Pedikelfehllage sicher ausschließen zu können, sollte man an dieser Stelle die Pedikelschrauben im a.-p.-Strahlengang einzeln orthograd einstellen (Punktprobe) und deren Lage zum Pedikel kontrollieren.

Nachdem die beiden Stäbe mit jeweils 2 Spezialbacken auf die Schanz-Schrauben geführt sind, werden die notwendigen Repositionsmanöver vorgenommen. Es besteht grundsätzlich die Möglichkeit der Lordosierung über die Schanzschrauben mit Hilfe der aufgesetzten Schraubenschlüssel, ferner die Möglichkeit der Distraktion über zwei Distraktoren, mit deren Hilfe die Schanzschrauben auf den Stäben gleitend auseinander geschoben werden. Welche der genannten Maßnahmen zuerst erfolgt, hängt vom Frakturtyp ab. Liegt eine keilförmige Verbildung des Wirbelkörpers vor oder eine 


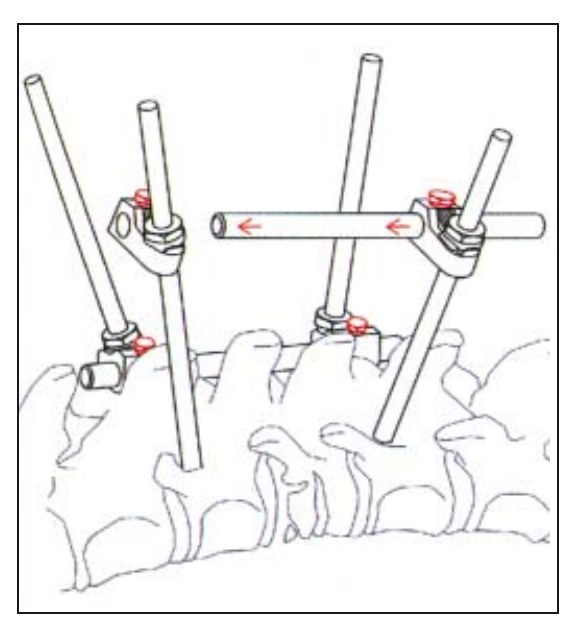

Abb. 9 Montage von Backen und Stäben.

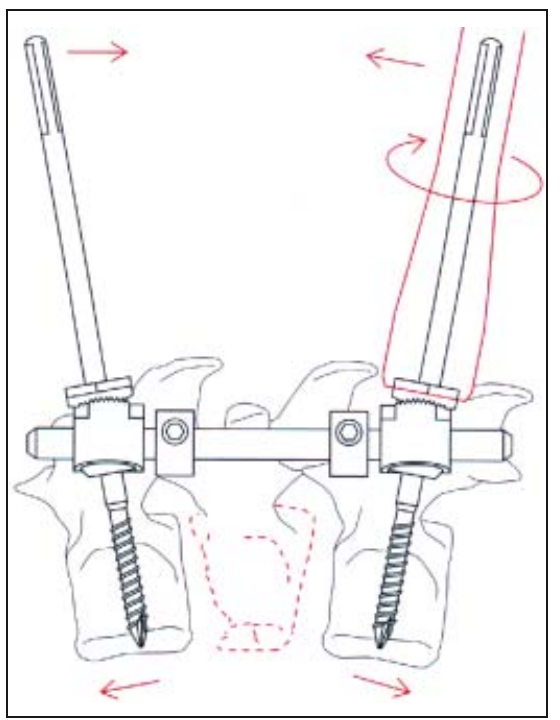

Abb.10 Reposition bei aufgesetztem Steckschlüssel/Lordosierung.
Distraktion, wird zuerst lordosiert, dann distrahiert, da nach primärer Distraktion die Spannung eine Lordosierung erschweren würde. Im Falle eines Berstungsbruches wird erst distrahiert, dann lordosiert. Dieses Vorgehen ist einzuhalten, da bei ungenügender Distraktion der Lordosierungsvorgang zu einem Drücken von Fragmenten in den Spinalkanal führen könnte.

Das Anbringen von Querverstrebungen zwischen den Stäben ist nur dann erforderlich, wenn Rotationsinstabilität besteht [3].

Nach abschließendem Überprüfen der Montage, Lagekontrolle der Schrauben und Stangen, Festziehen der Schrauben, werden die überstehenden SchanzSchraubenanteile mit einem Pinschneider gekürzt.

Vor dem Verschluss der Wunde wird diese gespült mit Kochsalzlösung, anschließend sorgsam debridiert, insbesondere die Muskulatur gegebenenfalls oberflächlich abgetragen, um avitales Gewebe zu entfernen.

Über jeweils einer Redondrainage werden die Fascia thoracolumbalis beiderseits sowie die Muskelfaszien an die intra- und supraspinösen Bänder angeheftet. Die Subkutanfaszie wird an diese Schicht genäht, um die Ausbildung von Hämatomen zu vermeiden.

Nach Einzelknopfnaht der Haut wird ein pelottierender Verband aufgelegt, um einer Hämatombildung vorzubeugen, der Patient auf den Rücken gelagert.

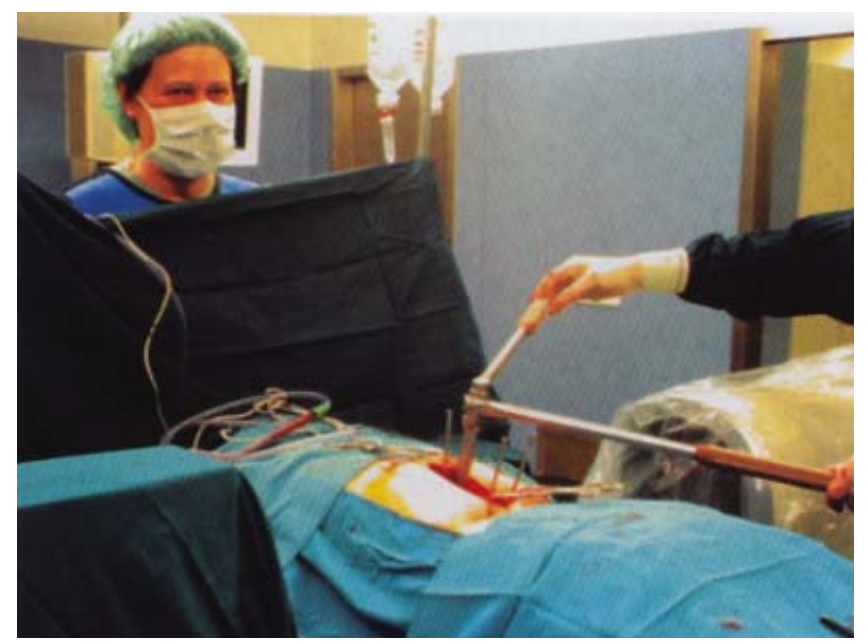

Im Anschluss an den Eingriff wird im OPSaal eine Röntgenaufnahme in 2 Ebenen angefertigt, um das Ergebnis zu dokumentieren.

Die Durchführung eines postoperativen Computertomogrammes ist dann erforderlich, wenn Unsicherheiten zur Pedikellage bestehen oder die Frage besteht, ob ein präoperativer spinaler Einstand gegebenenfalls beseitigt ist oder es einer Intervention von ventral bedarf. Vor allem im Bereich der BWS ist die Pedikellage allein mit konventionellen Röntgenbildern schwer zu beurteilen. Moderne Computertomographen können diese Fragen beantworten, selbst dann, wenn Stahlimplantate verwendet wurden.

\section{Nachsorge}

Die Drainagen werden am zweiten oder dritten Tag entfernt, anschließend noch für drei Tage die Mobilisation eingeschränkt, um einer Hämatombildung vorzubeugen. Die Stabilität nach dorsaler Stabilisierung ist in der Regel ausreichend, um eine Mobilisierung des Verletzten zu gewährleisten, auch dann, wenn eine zusätzliche ventrale Instrumentierung noch geplant ist.

Die Entfernung des Fixateur interne sollte nach Ablauf von 9 Monaten diskutiert werden. Sie ist anzustreben, da einerseits mitstabilisierte Nachbarsegmente freigegeben werden sollen, andererseits Ermüdungsbrüche des Materials vermieden werden können.

\section{Komplikationen}

Abb.11 Situs/Abschneiden der Pins.
Die häufigste spezifische Komplikation im Rahmen einer dorsalen Instrumentierung ist die Pedikelfehllage.

Die Schanz-Schraube kann die Pedikelwand perforieren, entsprechend bei kaudalem Durchtritt die Nervenwurzel irritieren, bei medialem Durchtritt in den Spinalkanal laufen und durch Blutungen neurologische Störungen hervorrufen. Bei lateralem Austreten leidet die Stabilität, der Schraubenhalt ist maßgeblich gemindert.

Eine intraoperative radiologische Kontrolle nach Einbringen der Pedikelschrauben lässt Fehllagen relativ zuverlässig erkennen, macht eine Korrektur möglich. Wenngleich abhängig von verschiedenen Arbeiten die Zahl der Pedikelfehllagen relativ hoch ist, bis $\mathrm{zu} \mathrm{40 \%} \mathrm{[4],} \mathrm{sind}$ 


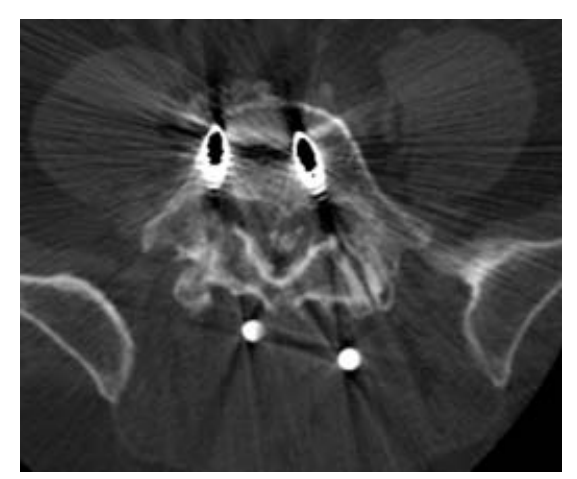

Abb.12 CT-Schnitt mit Abbildung Pedikelfehllage LWK 5.

schwer wiegende neurologische Komplikationen selten.

\section{Diskussion/Ausblick}

Die dorsale, transpedikuläre Instrumentierung ist als bekannteste, stabilste Fixierungstechnik an der Wirbelsäule ein weit verbreitetes Verfahren, das in der Lage ist, fast alle Frakturtypen der thorakolumbalen Wirbelsäule alleinig oder in Verbindung mit einer zusätzlichen ventralen Maßnahme zu stabilisieren. Korrektur und Stabilität werden in der Regel durch die dorsale Instrumentierung erreicht.
Eine ständige Verbesserung des Materials, insbesondere Erhöhung der Stabilität, verbunden mit einer Abnahme des Volumens und der Bauhöhe der Implantate, ermöglicht eine zunehmende Verringerung des Weichteiltraumas, welches mit dem Eingriff einhergeht.

Insbesondere die zugangsbedingte Schädigung der Muskulatur ist erheblich und kann durch schonende Implantationstechniken herabgesetzt werden. Diese minimal invasiven Techniken verlangen allerdings nach modifizierten Implantaten, die etwa ein Ablösen der Rückenmuskulatur von den Dornfortsätzen überflüssig macht.

Weitere wesentliche Verbesserungen gehen von den bildgebenden Verfahren aus. Die Qualität der Bildwandler beeinflusst maßgeblich die Ergebnisse vor allem durch die Darstellung der Pedikel, die im Bereich der mittleren und oberen BWS schwer ist.

Die CT-gestützte Navigation wird in absehbarer Zeit die Pedikulierung wesentlich vereinfachen, die Zielgenauigkeit verbessern und damit helfen, Pedikelschraubenfehllagen zu vermeiden, vor allem im Bereich der oberen Brustwirbelsäule.

\section{Literatur}

${ }^{1}$ Aebi. M. Operative Behandlung von Wirbelfrakturen - dorsale und ventrale Instrumentation. Op-Journal Nr. 2, August 1996; 182 187

${ }^{2}$ Bastian L, Knop C, Lange U. Transpedikuläre Implantation von Schrauben im Bereich der thorakolumbalen Wirbelsäule. Orthopäde 8 / 1999; 28: $693-702$

${ }^{3}$ Bastian L, Knop C, Lange U, Blauth M. Einfluss eines Querverbinders oder einer Cerclage auf die mechanische Stabilität des Fixateur interne. Orthopäde 1999; 28: 714-722

${ }^{4}$ Blauth M, Knop C, Bastian L. 4.Brust-und Lendenwirbelsäule. In: Tscherne Unfallchirurgie Wirbelsäule. 1. Auflage Springer, Berlin, Heidelberg, New York, S241-273

${ }^{5}$ Dick W. Internal Fixation of Thoracic and Lumbar Spine Fractures. Huber, Bern 1989

${ }^{6}$ Dickmann CA, Fessler RG, MacMillan M et al. Transpedicular screw-rod fixation of the lumbar spine: operative technique and outcome in 104 cases. J neurosurg 77: 860-870

${ }^{7}$ Harrington PR. Treatment of scoliosis. Correction and internal fixation by spine instrumentation. J Bone Joint Surg(AM) 1962; 44: $591-610$

${ }^{8}$ Knop C, Bastian L, Lange U et al. Transpedikuläre Spongiosaplastik am thorakolumbalen Übergang. Klinische, röntgenologische und klinische Ergebnisse. Orthopäde 8/ 1999; 28: $703-713$

${ }^{9}$ Kluger P, Gerner HJ. Das mechanische Prinzip des Fixateur externe zur dorsalen Stabilisierung der Brust-und Lendenwirbelsäule. Unfallchirurgie 1986; 12: $68-79$

${ }^{10}$ Magerl F, Aebi M, Gertzbein SD, Harms J, Nazarian S. A comprehensive classification of thoracic and lumbar injuries. Eur spine J 1994; 3: S. 184-201

${ }^{11}$ Roy-Camille R, Saillant G, Mazel C. Internal fixation of the lumbar spine with pedicle screw plating. Clin Orthop 203: 7-17

${ }^{12}$ Steinmann JC, Herkowitz HN et al. Spinal Pedicle Fixation. Confirmation of an image based technique for screw placement. Spine 18: $1856-1861$

\section{Dr. med. Hubertus Große-Leege} Oberarzt

Prof. Dr. med. Karl Heinrich Winker Direktor der Klinik für Unfallchirurgie

HELIOS-Klinikum Erfurt

Nordhäuser Str. 74

D-99089 Erfurt 